\title{
Photoacoustic microscopy of myocardial sheet architecture in unfixed and unstained mammalian hearts
}

Chi Zhang, Ya-Jian Cheng, Da-Kang Yao, Samuel Wickline, Lihong V. Wang

Chi Zhang, Ya-Jian Cheng, Da-Kang Yao, Samuel Wickline, Lihong V. Wang, "Photoacoustic microscopy of myocardial sheet architecture in unfixed and unstained mammalian hearts," Proc. SPIE 8223, Photons Plus Ultrasound: Imaging and Sensing 2012, 82232G (23 February 2012); doi: $10.1117 / 12.906208$ 


\title{
Photoacoustic microscopy of myocardial sheet architecture in unfixed and unstained mammalian hearts
}

\author{
Chi Zhang ${ }^{\mathrm{a}}$, Ya-Jian Cheng ${ }^{\mathrm{b}}$, Da-Kang Yao ${ }^{\mathrm{a}}$, Samuel Wickline ${ }^{\mathrm{b}}$, and Lihong V. Wang, \\ ${ }^{a}$ Optical Imaging Laboratory, Department of Biomedical Engineering, Washington University in St. \\ Louis, One Brookings Drive, St. Louis, MO 63130, USA; \\ ${ }^{\mathrm{b}}$ Cardiovascular Division, Washington University School of Medicine, Campus Box 8215,4320 \\ Forest Park Avenue, St. Louis, MO 63108, USA \\ * Corresponding author: lhwang@biomed.wustl.edu
}

\begin{abstract}
The laminar myocardial sheet architecture and its dynamic change play a key role in myocardial wall thickening. Histology, confocal optical microscopy (COM), and diffusion tensor MRI (DTI) have been used to unveil the structures and functions of the myocardial sheets. However, histology and COM require fixation, sectioning, and staining processes, which dehydrate and deform the sheet architecture. Although DTI can delineate sheet architecture nondestructively in viable hearts, it cannot provide cellular-level resolution. Here we show that photoacoustic microscopy (PAM), with high resolution $(\sim 1 \mu \mathrm{m})$ and label-free detection, is appropriate for imaging 3D myocardial architecture. Perfused half-split mouse hearts were also imaged by PAM in vitro without fixation, dehydration, nor staining. The laminar myocardial sheet architecture was clearly visualized within a $0.15 \mathrm{~mm}$ depth range. Two populations of oppositely signed sheet angles were observed. Therefore, PAM promises to access dynamic changes of myocardial architectures in ex vivo perfused-viable hearts.
\end{abstract}

Keywords: photoacoustic microscopy, myocardial sheet architecture, unfixed, unstained

\section{INTRODUCTION}

Studying the myocardial structure is critical to understanding lots of cardial functions, such as the contraction process and transmural wall thickening. Recently, the ventricular muscle fibers have been found to be organized in branching layers separated by cleavages, referred to as the "sheet architecture"[1],[2]. It has been demonstrated experimentally that the myocardial sheets provide a structural basis for systolic wall thickening ${ }^{[3]}$, and the systolic and diastolic shear deformation of ventrical wall are aligned along the sheets ${ }^{[4]}$. However, the detailed functional role of the sheet architecture is still poorly understood.

The studies on myocardial sheet architecture rely largely on the imaging technologies. Histology is the traditional way to image myocardial structures, but the sliced sections are usually deformed, which may result in inaccurate analysis. Confocal optical microscopy (COM) has been used to image the surface of the fixed heart tissue block, and the surface is then removed for imaging of deeper layers by $\mathrm{COM}^{[5]}$. Although the deformation induced by slicing can be avoided by this technique, the tissue still requires fixation and staining, which dehydrate and deform the sheet architecture. Diffusion tensor MRI (DTI) has been used to unveil the structures and functions of the myocardial sheets ${ }^{[2]}$. Although DTI can delineate sheet architecture nondestructively in viable hearts, it cannot provide cellular-level resolution (on the order of microns). Therefore, a high-resolution imaging technology is still required for visualizing the myocardial sheets in unfixed and unstained hearts.

Photoacoustic microscopy (PAM) is suitable for imaging myocardial sheet architecture for the following reasons. First, PAM can detect endogenous optical absorption contrast with a $100 \%$ sensitivity without labeling the tissue ${ }^{[6]}$. Second, PAM has high transverse resolution (down to $220 \mathrm{~nm}^{[7],[8]}$ ). Third, PAM can show the 3D sheet architecture with a larger penetration depth than most other optical imaging technologies ${ }^{[6]}$. So far, PAM has been mainly applied to structural and functional vascular imaging and melanoma tumor imaging in $v i v o^{[9]-[12]}$. In this paper, we realized label-free PAM of myocardial sheet architecture for the first time.

Photons Plus Ultrasound: Imaging and Sensing 2012, edited by Alexander A. Oraevsky, Lihong V. Wang, Proc. of SPIE Vol. 8223, 82232G · @ 2012 SPIE · CCC code: 1605-7422/12/\$18 - doi: 10.1117/12.906208 


\section{MATERIALS AND METHODS}

We used a high-resolution PAM system for heart imaging, as shown in Fig. $1^{[7],[8]}$. The system is briefly described here. A Nd:YVO4 laser generated pulses with a $532 \mathrm{~nm}$ wavelength and a $1.5 \mathrm{~ns}$ duration. The pulses were focused by a condenser lens and then coupled to a single-mode optical fiber. The light from the fiber was focused by a high-resolution optical objective. The focused pulse irradiated on the sample and generated photoacoustic waves, which were detected by a focused ultrasonic transducer ( $40 \mathrm{MHz}$ central frequency, $80 \%$ bandwidth). The photoacoustic signals were then amplified, digitized, and processed by a computer. Three-dimensional (3-D) imaging was achieved by two-dimensional (2-D) raster scanning of the objective and transducer while the depth was converted from the arrival time of the photoacoustic signals from each scanning point.

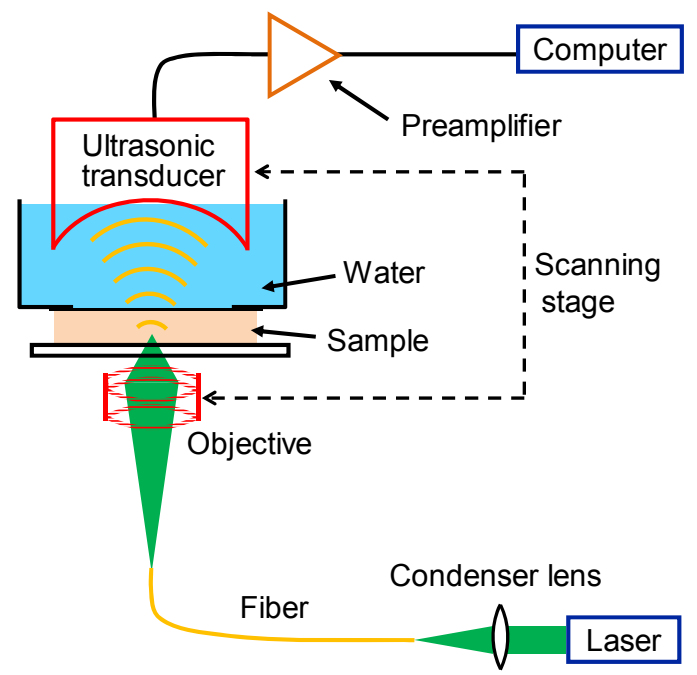

Figure 1. System schematic of photoacoustic microscopy.

The lateral resolution is determined by the light wavelength (fixed at $532 \mathrm{~nm}$ ) and the numerical aperture (NA) of the optical objective (changeable). The highest resolution achieved was $220 \mathrm{~nm}^{[7]}$. Here, in order to achieve a larger penetration depth, objectives with 0.60 and $0.32 \mathrm{NA}$ were used, providing lateral resolutions of $\sim 0.4 \mu \mathrm{m}$ and $\sim 0.8 \mu \mathrm{m}$, respectively. The axial resolution is determined by the bandwidth of the ultrasonic transducer ${ }^{[13]}$. However, by deconvolving the A-line data with the system impulse response, the axial resolution can be further improved ${ }^{[14]}$ and was estimated to be $\sim 20 \mu \mathrm{m}$ here.

\section{RESULTS AND DISCUSSION}

An excised and half-split mouse heart was imaged by PAM. The heart was blood-perfused to avoid the strong signals from hemoglobin, but the heart was unfixed and unstained and thereby undistorted. Fig. 2(a) shows a picture of the halfsplit heart. The square region is a part of the LV free wall (FW) and will be imaged by PAM. The PAM image of the FW is shown in Fig. 2(b). The image was a maximum amplitude projection along the depth direction from $10 \mu \mathrm{m}$ to $70 \mu \mathrm{m}$ in depth. The close-up image of the marked region in Fig. 2(b) is shown in Fig. 2(c), where the branching sheets are clearly shown. Two populations of oppositely signed sheet angles were observed. The boundaries of the sheets were extracted. 

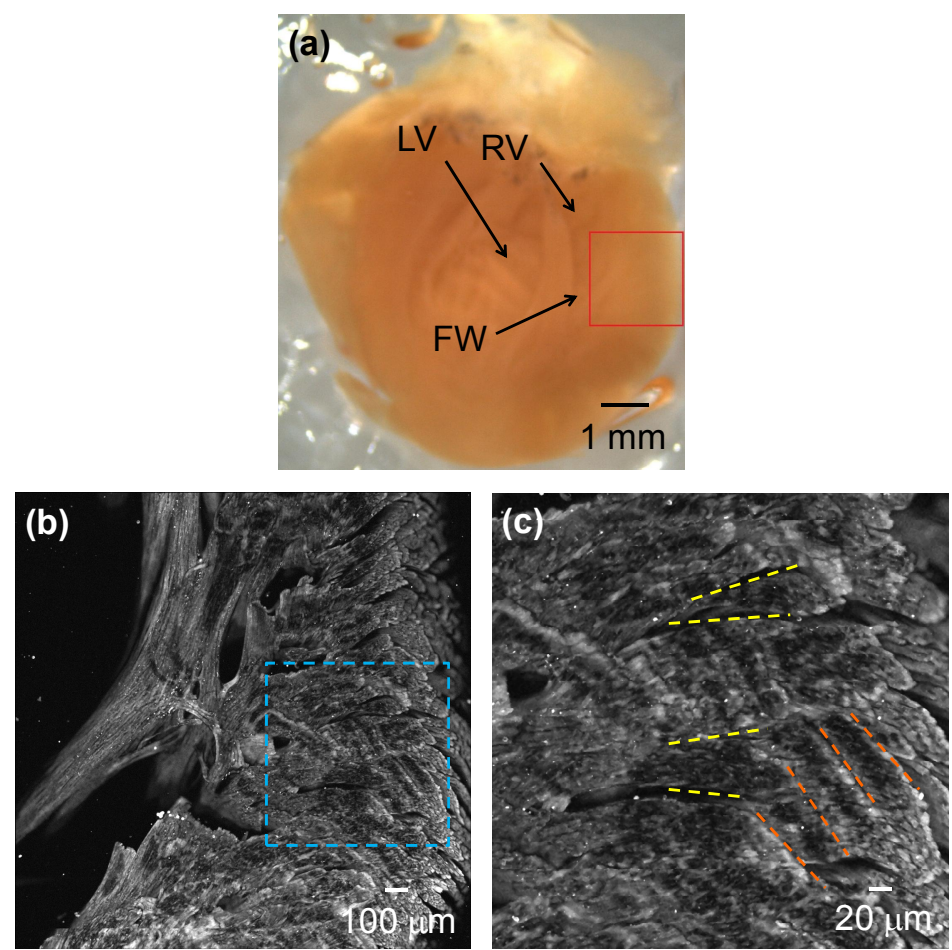

0

\section{Optical absorption}

1

Figure 2. A blood-perfused half-split mouse heart (unfixed and unstained). (a) A picture of the heart. The square region will be imaged by PAM. LV, left ventricle; RV, right ventricle; FW, free wall. (b) PAM of the FW. (c) Close-up image of the marked region in (b). The boundaries of the branching sheets are extracted.

The 3-D sheet architecture of the same heart was shown by Video 3. The heart was scanned twice, focusing at $40 \mu \mathrm{m}$ and $100 \mu \mathrm{m}$ deep, respectively. Video 3 is a movie of 100 image stacks in the same area as Fig. 2(b) scanning from 0 $\mathrm{mm}$ to $0.15 \mathrm{~mm}$ in depth. How the sheets develop along the depth direction was visualized. Therefore, PAM can show accurate 3-D myocardium structure in unfixed and unstained hearts.

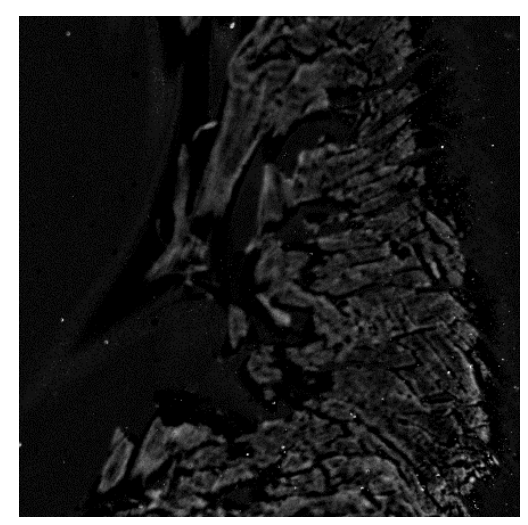

Video 3. A movie of image stacks in the same area as Fig. 2(b) scanning from $0 \mathrm{~mm}$ to $0.15 \mathrm{~mm}$ in depth. http://dx.doi.org/10.1117/12.906208.1 
We expect to further develop PAM for accessing dynamic changes of myocardial architectures in ex vivo perfused-viable hearts. The heart can be arrested in diastole and systole in sequence during which the heart is imaged by PAM. The functional role of the myocardial sheets will be analyzed. For this study, we need to improve the penetration depth and axial resolution of $\mathrm{PAM}^{[15]}$, so the heart doesn't need to be split into halves, and the function of the heart can be well preserved. The imaging speed should also be enhanced ${ }^{[16],[17]}$ because the ex vivo heart can be maintained viable for only a few hours.

\section{CONCLUSIONS}

In summary, we realized label-free PAM of myocardial sheet architecture for the first time. With high spatial resolution and endogenous optical absorption contrast, label-free PAM reveals the myocardial sheet architectures. Without unwanted artifacts introduced by the tissue preparation as used in histology, PAM can provide accurate functional parameters. With further technical developments, PAM promises to assess dynamic changes of myocardial architectures in ex vivo perfused-viable hearts.

\section{ACKNOWLEDGMENTS}

This work was sponsored in part by National Institutes of Health grants R01 EB000712, R01 EB008085, R01 CA134539, U54 CA136398, R01 CA157277, and 5P60 DK02057933. L.W. has a financial interest in Microphotoacoustics, Inc. and Endra, Inc., which, however, did not support this work.

\section{REFERENCES}

[1] LeGrice, I. J., Smaill, B. H., Chai, L. Z., Edgar, S. G., Gavin, J. B. and Hunter, P. J., "Laminar structure of the heart: ventricular myocyte arrangement and connective tissue architecture in the dog," Am. J. Physiol. Heart Circ. Physiol. 269, H571-H582 (1995).

[2] Chen, J., Liu, W., Zhang, H., Lacy, L., Yang, X., Song, S.-K., Wickline, S. A. and Yu, X., "Regional ventricular wall thickening reflects changes in cardiac fiber and sheet structure during contraction: quantification with diffusion tensor MRI,” Am. J. Physiol. Heart Circ. Physiol. 289, H1898-H1907 (2005).

[3] LeGrice, I. J., Takayama, Y. and Covell, J. W. "Transverse shear along myocardial cleavage planes provides a mechanism for normal systolic wall thickening," Circ. Res. 77, 182-193 (1995).

[4] Costa, K. D., Takayama, Y., McCulloch, A. D. and Covell, J. W. "Laminar fiber architecture and three-dimensional systolic mechanics in canine ventricular myocardium," Am. J. Physiol. Heart Circ. Physiol. 276, H595-H607 (1999).

[5] Pope, A. J., Sands, G. B., Smaill, B. H. and LeGrice, I. J., "Three-dimensional transmural organization of perimysial collagen in the heart," Am. J. Physiol. Heart Circ. Physiol. 295, H1243-H1252 (2008).

[6] Wang, L. V., "Multiscale photoacoustic microscopy and computed tomography," Nat. Photon. 3(9), 503-509 (2009).

[7] Zhang, C., Maslov, K. and Wang, L. V., "Subwavelength-resolution label-free photoacoustic microscopy of optical absorption in vivo," Opt. Lett. 35(19), 3195-3197 (2010).

[8] Zhang, C., Maslov, K. and Wang, L. V., "Subwavelength-resolution photoacoustic microscopy for label-free detection of optical absorption in vivo", Proc. SPIE 7899, 78990L (2011).

[9] Zhang, H. F., Maslov, K., Stoica, G. and Wang, L. V., "Functional photoacoustic microscopy for high-resolution and noninvasive in vivo imaging," Nat. Biotech. 24, 848-851 (2006).

[10] Hu, S. and Wang, L. V., "Photoacoustic imaging and characterization of the microvasculature," J. Biomed. Opt. 15(1), 011101 (2010).

[11] Wang, Y., Maslov, K., Zhang, Y., Hu, S., Yang, L., Xia, Y., Liu, J. and Wang, L. V., "Fiber-laser-based photoacoustic microscopy and melanoma cell detection," J.Biomed. Opt. 16, 011014 (2011).

[12] Wang, Y., Hu, S., Maslov, K., Zhang, Y., Xia, Y. and Wang, L. V., "In vivo integrated photoacoustic and confocal microscopy of hemoglobin oxygen saturation and oxygen partial pressure," Opt. Lett. 36(7), 1029-1031 (2011).

[13] Ku, G., Maslov, K., Li, L. and Wang, L. V., "Photoacoustic microscopy with 2- $\mu \mathrm{m}$ transverse resolution," J. Biomed. Opt. 15, 021302 (2010). 
[14] Zhang, C., Maslov, K., Hu, S., Chen, R., Zhou, Q, Shung, K. K. and Wang, L. V., "Reflection-mode submicronresolution in vivo photoacoustic microscopy," J. Biomed. Opt., accepted for publication.

[15] Xie, Z., Chen, S.-L., Ling, T., Guo, L. J., Carson, P. L. and Wang, X., "Pure optical photoacoustic microscopy," Opt. Express 19(10), 9027-9034 (2011).

[16] Rao, B., Maslov, K., Danielli, A., Chen, R., Shung, K. K., Zhou, Q. and Wang, L. V., "Real-time four-dimensional optical-resolution photoacoustic microscopy with Au nanoparticle-assisted subdiffraction-limit resolution," Opt. Lett. 36(7), 1137-1139 (2011).

[17] Wang, L., Maslov, K., Yao, J., Rao, B. and Wang, L. V., "Fast voice-coil scanning optical-resolution photoacoustic microscopy," Opt. Lett. 36(2), 139-141 (2011). 